\title{
Nonrestorative sleep scale: a reliable and valid short form of the traditional Chinese version
}

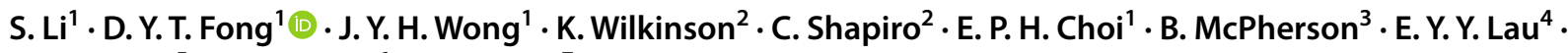 \\ C. L. K. Lam $^{5}$ L. X. Huang ${ }^{6}$ M. S. M. Ip
}

Accepted: 5 May 2020 / Published online: 16 May 2020

(c) The Author(s) 2020

\begin{abstract}
Purpose Previous research has suggested the essential unidimensionality of the 12-item traditional Chinese version of the Nonrestorative Sleep Scale (NRSS). This study aimed to develop a short form of the traditional Chinese version of the NRSS without compromising its reliability and validity.

Methods Data were collected from 2 cross-sectional studies with identical target groups of adults residing in Hong Kong. An iterative Wald test was used to assess differential item functioning by gender. Based on the generalized partial credit model, we first obtained a shortened version such that further shortening would result in substantial sacrifice of test information and standard error of measurement. Another shortened version was obtained by the optimal test assembly (OTA). The two shortened versions were compared for test information, Cronbach's alpha, and convergent validity.

Results Data from a total of 404 Chinese adults (60.0\% female) who had completed the Chinese NRSS were gathered. All items were invariant by gender. A 6-item version was obtained beyond which the test performance substantially deteriorated, and a 9-item version was obtained by OTA. The 9-item version performed better than the 6-item version in test information and convergent validity. It had discrimination and difficulty indices ranging from 0.44 to 2.23 and -7.58 to 2.13 , respectively, and retained $92 \%$ of the test information of the original 12 -item version.
\end{abstract}

Conclusion The 9-item Chinese NRSS is a reliable and valid tool to measure nonrestorative sleep for epidemiological studies.

Keywords Item response theory $\cdot$ Item selection $\cdot$ Nonrestorative sleep $\cdot$ Optimal test assembly $\cdot$ Reliability $\cdot$ Validity

Electronic supplementary material The online version of this article (https://doi.org/10.1007/s11136-020-02523-4) contains supplementary material, which is available to authorized users.

D. Y. T. Fong

dytfong@hku.hk

1 School of Nursing, The University of Hong Kong, 21 Sassoon Road, Hong Kong, China

2 Department of Psychiatry, University of Toronto, 399 Bathurst Street, Toronto, Canada

3 Division of Speech and Hearing Sciences, The University of Hong Kong, Pokfulam Road, Hong Kong, China

4 Department of Psychology, The Education University of Hong Kong, 10 Lo Ping Road, Hong Kong, China

5 Department of Family Medicine and Primary Care, The University of Hong Kong, 21 Sassoon Road, Hong Kong, China

6 Department of Mechanical Engineering, The University of Hong Kong, Pokfulam Road, Hong Kong, China

7 Department of Medicine, The University of Hong Kong, 21 Sassoon Road, Hong Kong, China

\section{Introduction}

Nonrestorative sleep (NRS) refers to the subjective feeling of being restless or un-refreshed even with normal sleep duration $[1,2]$. Estimates of the prevalence of NRS may range from 2.4 to $43 \%$ in the general adult population across different ethnic groups or using different instruments [3-6], while its prevalence in Hong Kong has been found to be $8.1 \%$ [7]. NRS may cause health problems such as fatigue during the day, resulting in decreased daytime function and performance, and increased occupational and nonoccupational accidents [8]. In addition, reduced psychological wellbeing, mental problems, and chronic disease have also been observed to be strongly associated with NRS [7, 9-11]. NRS sometimes does and sometimes does not coexist with other sleep problems, and hence has been viewed as a discrete treatment target in recent years [12].

In order to properly assess NRS, Wilkinson and Shapiro developed the first appropriate assessment instrument, 
the Nonrestorative Sleep Scale (NRSS) [13]. The original English version of the NRSS comprised 12 items [13], covering 4 domains: refreshment from sleep, physical/medical symptoms of NRS, daytime functioning, and affective symptoms of NRS. This 12-item NRSS has been translated into traditional Chinese script, with both the original English and traditional Chinese versions demonstrating a valid, reliable 4-factor structure for assessing NRS [13, 14]. Moreover, the traditional Chinese NRSS showed essential unidimensionality in a bifactor analysis [14]. Although the 12-item NRSS is well established, whether it can be shortened, in order to reduce response burden and increase respondent acceptability, has not been explored.

The traditional classical test theory approach assumes constant reliability across all scores, an assumption that is often violated in reality [15]. Moreover, the scale properties obtained on this basis are usually sample dependent, which implies the need to test these properties again in other populations [15]. In contrast, modern item response theory (IRT) focuses on the item level and is sample independent. Moreover, IRT makes it possible to select items that offer more information concerning the measured trait. In this way, a well-performing shortened scale that retains an adequate amount of information along with the precision of the original scale can be obtained [16]. However, the item performance of the NRSS has not been examined using IRT.

Therefore, this study aimed to use develop a short version of the Chinese NRSS to facilitate the quick and efficient assessment of NRS.

\section{Methods}

\section{Participants}

Individuals who were 18 years old or above and were able to read traditional Chinese or communicate in Cantonese were recruited. Those who were not willing to participate or had difficulty understanding the study procedures were excluded. In addition, people who were taking medication for sleep disorders or had psychiatric illnesses were excluded. To conduct a generalized partial credit model (GPCM) for the analysis of polytomous responses, a sample size of at least 250 respondents is acceptable [17]; hence, a sample of 250 respondents or more was targeted, and ultimately a total of 404 subjects were recruited across 2 studies.

\section{Procedures}

A total of 404 people who had completed the 12-item Chinese NRSS were gathered from 2 studies that had the same eligibility criteria. The first was a cross-sectional study that recruited 120 participants by telephone and home visit between September 2016 and July 2017 [14]. The second was a household survey that recruited 284 participants between May 2018 and March 2019. Potential participants were identified from a list of household addresses maintained by the Hong Kong Census and Statistics Department. The records of household addresses were grouped into quarters, and a random sample of the quarters was obtained by systematic sampling design with fixed sampling intervals and nonrepetitive random numbers; all households residing in the selected quarters were covered in the survey. In both studies, when there was more than one eligible person living in the household, the person closest to their next birthday was recruited. Ethics approval for both studies was obtained from the Institutional Review Board of the University of Hong Kong/Hospital Authority Hong Kong West Cluster (Ref nos. UW16-326 and UW 17-011).

\section{Measurements}

\section{The nonrestorative sleep scale (NRSS)}

The 12-item NRSS has 6 items that require reverse coding. Moreover, 10 items require responses on a 1-10 scale with adjacent responses scored as $1-5$, that is, responses 1 and 2 scored as 1 , responses 3 and 4 scored as 2 , etc. The other 2 items require responses scored on a 1-5 scale. A higher total scale score corresponds to less NRS [13].

\section{The pittsburgh sleep quality index (PSQI)}

The PSQI was developed to evaluate sleep quality during the past month [18]. The items are grouped into 7 components, including subjective sleep quality, sleep latency, sleep duration, habitual sleep efficiency, sleep disturbance, use of sleeping medications, and daytime dysfunction, with each rated on a $0-3$ scale [18]. The total score ranges from 0 to 21, with a higher score indicating poorer sleep quality [19]. Previous studies used PSQI to test the convergent validity with NRSS [13, 14].

\section{Statistical analysis}

The scale scores were summarized with descriptive statistics. NRSS item reduction was done by examining item characteristics using IRT analysis.

We first assessed the two assumptions of IRT: unidimensionality and local independence [20]. Unidimensionality for the 12-item NRSS was previously shown in a bifactor model [14]. Nevertheless, we retested this assumption in our sample using minimum residual factoring of the polychoric correlation matrix exploratory factor analysis (EFA) [21]; specifically, we assessed Kaiser-Meyer-Olkin (KMO) and Bartlett's test of sphericity values to confirm the 
appropriateness of the EFA [22]. Then, the number of factors was identified by assessing the scree plot [23]. Unidimensionality was accepted if the first factor explained more than $20-40 \%$ of variance $[24,25]$ and the ratio of the eigenvalues of the first to the second unrotated factor was greater than 3 [20]. Local independence refers to independence among responses across items conditioned on the corresponding latent trait [26]; its presence was accepted if the residual correlations for the items were smaller than 0.25 [27]. After confirming unidimensionality and local independence, we fitted a GPCM.

Under the GPCM, we obtained the discrimination parameter $\left(a_{i}\right)$ and the difficulty parameters $\left(b_{i}\right)$ for each item. A higher discrimination parameter value indicates greater ability of the corresponding item to differentiate respondents at different trait levels. In this study, ability refers to the NRS level. The discrimination parameter often ranges from 0.5 to 2.5 , and items with values smaller than 0.4 are recommended for removal [28]. As for the number of difficulty parameters, it corresponds to the number of response categories minus 1. Under the GPCM, the difficulty parameter refers to the latent trait level where the probabilities of endorsing the two adjacent response categories are the same [29]. Item characteristic curves (ICCs) were also obtained, with the curve steepness reflecting the discrimination level: greater steepness demonstrated greater discrimination ability [30].

To examine differential item functioning (DIF) by gender, that is, to determine whether there were items that were responded differently by male and female participants even when they had the same trait level [31], similarity of slopes and intercepts by gender was tested using an iterative Wald approach, with a significant $p$-value indicating DIF [32]. Specifically, the Wald-2 approach was used to identify the anchor items, that is, items showing invariance across groups. To better control type 1 error rate, we also adopted the MaxA5 method, which uses 5 items with the largest discrimination parameters as the anchor items [33], before the Wald-1 approach was iteratively used to test for DIF items [32]. The female group was set as the reference group and the male group was set as the focal group.

In addition, we also obtained item information, test information and average standard error (SE). Test information, which is the sum of the item information for all items, provides evidence on how accurately the test estimates a latent trait over the entire range of trait levels. The more information at a particular trait level provided by the test, the higher the precision of ability estimation, and the higher the reliability [34]. Test information was obtained over the entire range of latent trait levels $(\theta)$, as well as over the common range of $(-3,3)$ used to avoid potentially inflated information due to the presence of extremely able/unable participants [35]. The SE refers to the standard error of latent trait estimates, which indicates the amount of information unexplained by the items being considered. It is independent of the distribution of scores in the obtained sample [36]. It was taken as the average of SE at each latent trait level in the study.

We tried two item selection approaches that had been adopted in the literature. First, the item reduction process was initiated by removing, first, items with discrimination $<0.4$ and then items that showed DIF by gender. The process continued with the assessment of test information and SE of measurement. Specifically, first, the item that carried the lowest item information was removed, and test information and SE were assessed. Then, the item that carried the next-lowest item information was removed and test information and SE again assessed. We continued the removal of items until there was a relatively substantial reduction in test information and increase in SE. Finally, this shortened NRSS was tested again for item discrimination and difficulty using IRT.

Second, optimal test assembly (OTA) was adopted, after excluding the items showing DIF. For each fixed number of items between 3 and 12, the set of NRSS items that maximized the total test information over five anchor points $(\theta$ : $-3,-1,0,1,3)$ based on GPCM was first obtained using the branch-and-bound algorithm [37]. Then, the shortened version was taken as the smallest set of items that satisfied three criteria: (1) the correlation between the factor scores (as well as summed scores) of the shortened version and those of the 12 -item version should be at least 0.95 ; (2) the convergent validity correlation between the factor scores (as well as summed scores) and PSQI should be within a tolerance of 0.05 when compared with that of the 12-item version; and (3) the Cronbach's alpha should be at least $95 \%$ of that of the 12-item version.

The obtained shortened versions were compared with the original 12-item version in terms of test information, Cronbach's alpha, and convergent validity.

The EFA was conducted with the package "psych" [38], while DIF was tested with the package "mirt" [39]. The R package "ltm" was used to run the OTA procedure [40]. The OTA was procedure was implemented through the package "IpSolveAPI" [41]. All of the packages were run in RStudio 1.1.383. SEs for each latent trait level were obtained in IRTPRO (4.2 Student version).

\section{Results}

\section{Participant demographic characteristics}

The combined sample had 162 male and 242 female respondents. Their average age was 45 years (standard deviation: 17 ; range: $18-88)$. In all, 38 participants $(9.4 \%)$ had primary education or below, $213(52.7 \%)$ had secondary 
education, and 151 (37.4\%) had a bachelor's degree or above. There were 238 (58.9\%) workers, 60 (14.9\%) homemakers, 31 (7.7\%) students, 68 (16.8\%) retired participants, and $6(1.5 \%)$ job-seeking participants. All participants completed the 12 -item NRSS without any missing values.

\section{Checking IRT assumptions for the full NRSS}

The KMO statistic was 0.86 and Bartlett's test was statistically significant $(p<0.001)$. The EFA identified one factor, with the eigenvalue of the first factor (4.6) substantially larger than those of the next 2 factors (1.3 and 0.7, respectively). The first rotated factor explained $38 \%$ of total variance, much higher than the second factor, which explained only $11 \%$; therefore, the 12 -item test could be regarded as essentially unidimensional. Moreover, the residual correlations among the 12 items ranged from 0.21 to 0.22 , all smaller than 0.25 ; hence, the local independence assumption was also met.

\section{NRSS item properties and selection}

Table 1 shows the values of the discrimination and difficulty parameters for the 12 items of NRSS. Discrimination for the 12 items ranged from 0.33 to 2.10 . Items with the highest 5 discrimination parameters were Q10 $(a=2.10)$, Q1 $(a=1.74), \mathrm{Q} 2(a=1.39), \mathrm{Q} 9(a=1.36)$, and Q3 $(a=1.24)$. Appendix 1 in shows the ICCs of all 12 NRSS items. Table 1 also shows the total information of each item which lay between 1.29 and 8.41. Item 5 was removed due to the small discrimination parameter.
Using the iterative Wald test approach, the Wald-2 test (all-others-as-anchors model) revealed DIF by gender only in Q8. However, after setting the five items with the largest discrimination parameters as anchor items in Wald-1 test, Q8 had a $p$ value of 0.061 and thus was concluded to be invariant by gender.

Appendix 2 graphically shows changes in test information and SE as more items were removed. When Q5, Q11, Q4, Q7, Q6, and Q12 were removed, the additionally removed Q8 resulted in greater increase of SE and decrease of test information when compared with the original 12-item scale. Therefore, we retained 6 items-Q1, Q2, Q3, Q8, Q9, and $\mathrm{Q} 10$. The OTA procedure and decision rules removed Q4, Q5, and Q11, which had the smallest discrimination parameters and item information, resulting in a 9-item scale-Q1, Q2, Q3, Q6, Q7, Q8, Q9, Q10, and Q12. The 9-item scale had a Cronbach's alpha value of 0.835 . The correlation between the summed scores of the 9-item and 12-item versions was 0.960 , while that between the factor scores was 0.995 . The correlations between the PSQI and the 9 -item summed and factor scores were 0.975 and 0.966 , respectively.

\section{Testing the shortened traditional Chinese NRSS scales}

IRT analysis on the 6-item Chinese NRSS showed that all items had discrimination parameters ranging from 1.26 to 2.22 , while difficulty parameters ranged from -3.13 to 2.13 . The discrimination parameters and difficulty parameters for

Table 1 The discrimination, difficulty, and item information of the 12 Chinese NRSS items

\begin{tabular}{|c|c|c|c|c|c|c|}
\hline \multirow[t]{2}{*}{ Items } & \multirow[b]{2}{*}{$a_{i}$} & \multicolumn{4}{|c|}{ Difficulty parameters $\left(b_{i j}\right)$} & \multirow{2}{*}{$\begin{array}{l}\text { Item } \\
\text { informa- } \\
\text { tion }\end{array}$} \\
\hline & & $b_{i 1}$ & $b_{i 2}$ & $b_{i 3}$ & $b_{i 4}$ & \\
\hline Q1: ...rate the quality of your sleep & 1.74 & -2.12 & -1.20 & -0.12 & 2.14 & 6.94 \\
\hline Q2: ...sleep is restoring or refreshing? & 1.39 & -3.21 & -1.70 & -0.50 & 1.55 & 5.57 \\
\hline Q3: ...felt rested if you've slept for your usual amount of time? & 1.24 & -2.08 & -0.84 & -0.27 & 1.76 & 4.97 \\
\hline Q4: ...had physical sensations or unusual feelings? & 0.43 & -5.26 & -1.83 & 0.03 & -0.54 & 1.66 \\
\hline $\begin{array}{l}\text { Q5: ... one or more of the following: headaches, body pain, numb- } \\
\text { ness, or tingling...? }\end{array}$ & 0.33 & -0.72 & -4.68 & -1.58 & -2.87 & 1.29 \\
\hline Q6: physical or medical problems are dragging you down? & 0.65 & -4.94 & -1.41 & 0.73 & -0.61 & 2.56 \\
\hline Q7: ... have a sense of panic, or physical symptoms of panic...? & 0.53 & -6.42 & -1.67 & -0.81 & -1.74 & 2.06 \\
\hline Q8: ...memory and concentration...? & 1.12 & -2.07 & -1.65 & -0.42 & 2.20 & 4.49 \\
\hline Q9: ...level of daytime energy? & 1.36 & -2.50 & -1.73 & -0.44 & 2.14 & 5.45 \\
\hline Q10: ....alert during the daytime? & 2.10 & -2.20 & -1.77 & -0.74 & 1.46 & 8.41 \\
\hline Q11: ...depressed or down if you didn't sleep well...? & 0.40 & -5.64 & -1.29 & 0.37 & -0.18 & 1.54 \\
\hline Q12: ...irritable or "gotten the blahs" if you didn't sleep well & 0.98 & -2.50 & -2.88 & -1.07 & -0.09 & 3.90 \\
\hline
\end{tabular}

$a_{i}$ the discrimination parameter, i.e., the ability of an item to discriminate different levels of nonrestorative sleep, $b_{i j}$ difficulty parameter, represents the latent trait level of choosing response $j$ instead of $j-1$ of item $i$ 


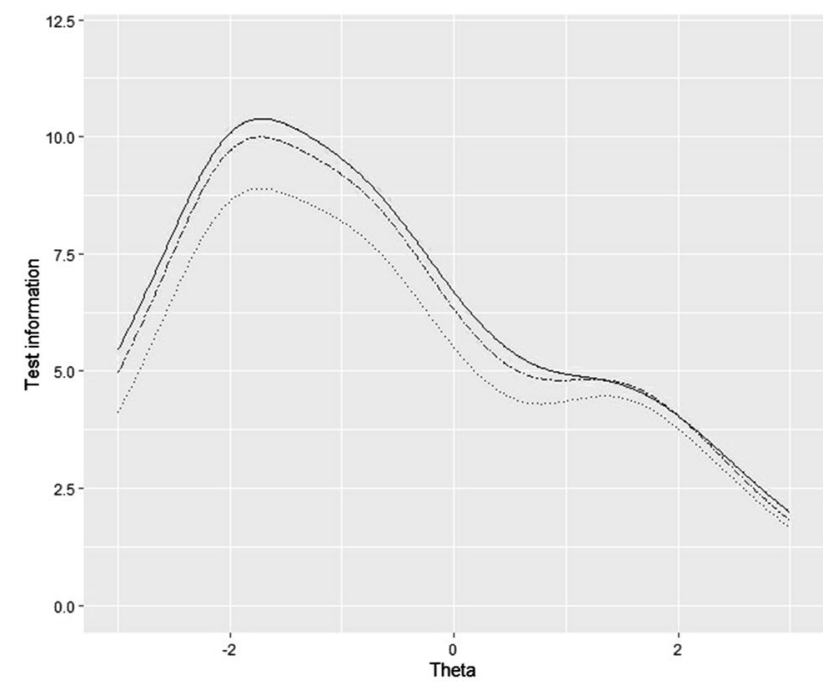

Fig. 1 Test information curves of the original 12-item NRSS (solid curve), the 9-item NRSS (longdash curve), and 6-item NRSS (dotted curve)

Table 2 Comparison on test information, Cronbach's alpha, and convergent validity of different versions of NRSS

\begin{tabular}{lccc}
\hline & 12 items & 9 items & 6 items \\
\hline Test information $^{\mathrm{a}}$ & 48.87 & 45.07 & 38.28 \\
${\text { Test information }(-3,3)^{\mathrm{b}}}$ & 40.09 & 38.27 & 33.64 \\
Cronbach's alpha $_{\text {Convergent validity }}$ & 0.834 & 0.835 & 0.843 \\
\hline
\end{tabular}

${ }^{a}$ Test information over the entire latent trait level

${ }^{\mathrm{b}}$ Test information over the latent trait level $(\theta)$ of $(-3,3)$

the 9-item scale ranged from 0.44 to 2.23 , and from -7.58 to 2.13 , respectively.

Figure 1 shows the test information for the original 12-item, the 6-item, and the 9-item NRSS versions between NRS level $(\theta)$ range of $(-3,3)$. The test information of the 9 -item version closely resembled that of the original 12-item version. Table 2 compares the test information, Cronbach's alpha, and convergent validity with PSQI of the different versions. The 6-item and the 9-item versions, respectively, kept $78 \%$ and $92 \%$ of the test information over the entire $\theta$ range of the original 12 -item scale, and kept $84 \%$ and $95 \%$ between $\theta(-3,3)$. The Cronbach's alpha was quite similar across all versions. The 9-item version had higher convergent validity and test information, and kept at least one item for each of the four domains in the 12-item version. Therefore, the 9-item version performed better than the 6-item version.

\section{Discussion}

This study has made the first attempt to develop a short form NRSS for assessing NRS. Using the IRT and OTA methodology, we obtained a 9-item scale that kept at least one item from each of the 4 hypothesized domains of the NRSS. All the retained items had relatively high discriminative power, and the shortened scale had test information closely resembling that of the original 12 -item scale.

Higher discrimination of an item means that the probability of choosing a particular response for that item will increase more rapidly as the ability level of a respondent increases [42]. Baker proposed an interpretation of discrimination values as follows: $0=$ nondiscriminative; $0.01-0.34=$ very low; $0.35-0.64=$ low; $0.65-1.34=$ moderate; $1.35-1.69=$ high; $>1.70=$ very high [30]. The discrimination parameters for the 9 retained items ranged from 0.44 to 2.23 , corresponding to low-to-high discrimination for assessment of NRS. However, we kept the item with the discrimination of 0.44 as a cut off value of 0.4 has been suggested.

Using the Wald-2 test, gender-related DIF was detected on item Q8, which asked "How is your memory and concentration during the daytime?," but no DIF was detected in the second stage by Wald- 1 test. The Wald- 2 is known to be more likely to inflate Type I error rate [43]. This study adopted the iterative Wald test approach, which was shown, in simulation study, to outperform the Wald- 1 and Wald-2 tests when they were used individually, in that it had lower Type I and Type II error rates [32, 33]. Nevertheless, since the $p$ value of 0.061 was marginal, more data and studies are worth pursuing.

Based on OTA with GPCM, items Q5, Q11, and Q4 were removed. These three items carried low information and were all negatively worded. Previous study has indicated that question phrasing methods may impact the performance of a questionnaire with Chinese respondents [44]. In particular, Q5 asked about symptoms such as "headaches, body pain, numbness, tingling, nausea, racing heart/palpitations, sore throat, frequent cough"; however, the extent to which such specific symptoms are associated with NRS has not been well determined, and more studies are necessary to confirm such associations, if any. Q11 asked "Do you feel depressed or down if you didn't sleep well the night before?.” Depression or depressed feeling may take an extended time to develop and would be unlikely to develop merely if one had not slept well recently, ceteris paribus. Q4 asked "Have you had physical sensations or unusual feelings in your body that you couldn't identify?." Its low information value is indeed consistent with what we observed in our post-test debriefing, when some people stated they were unsure how one could 
have physical sensations or unusual feelings that could not be identified.

The 9-item version retained $92 \%$ and $95 \%$ of the test information of the original 12-item version over the entire NRS level range and the range of $(-3,3)$, respectively. Their test information functions were very close to each other and were the highest over around the same range of the latent trait. These findings demonstrate that adoption of the shortened 9-item version would not substantially sacrifice precision or reliability.

Recently, OTA has demonstrated value in developing short forms of PROs [45, 46]. It offers an appealing solution for determining a replicable and reproducible short form. Nevertheless, despite our efforts in conducting a rigorous study, there are several limitations worth noting and also worth addressing in future work. First, although our study size was adequate for typical IRT analysis, a larger population-based study would be desirable to establish Chinese NRSS short form norms that can facilitate the interpretation of the tool. Second, DIF analysis for other participant groups, such as language groups, should also be done, for cross-cultural comparison. Third, the multidimensional IRT has been made available to allow IRT to be conducted on multidimensional scales. Since the traditional Chinese NRSS is essentially unidimensional, we did not pursue multidimensional IRT. Lastly, the reliability and validity of the 9-item scale would preferably be further examined in an independent sample.

Conclusively, nevertheless, the 9-item traditional Chinese NRSS is a reliable and valid tool for assessing NRS. The short form allows more efficient assessment by healthcare professionals, researchers, and the public.

Acknowledegements We would like to thank Editage (www.edita ge.com) for English language editing.

Funding This work was supported by the Health and Medical Research Fund [Grant No. 14150801], the Food and Health Bureau, Hong Kong Special Administrative Region, and the Seed Fund for Basic Research [Grant No. 201511159061] from The University of Hong Kong.

\section{Compliance with ethical standards}

Conflict of interest The authors declare no conflict of interest.

Informed consent Informed consent was obtained from all individual participants included in the study.

Ethical approval All procedures performed in studies involving human participants were in accordance with the ethical standards of the institutional and/or national research committee and with the 1964 Helsinki declaration and its later amendments or comparable ethical standards.

Open Access This article is licensed under a Creative Commons Attribution 4.0 International License, which permits use, sharing, adaptation, distribution and reproduction in any medium or format, as long as you give appropriate credit to the original author(s) and the source, provide a link to the Creative Commons licence, and indicate if changes were made. The images or other third party material in this article are included in the article's Creative Commons licence, unless indicated otherwise in a credit line to the material. If material is not included in the article's Creative Commons licence and your intended use is not permitted by statutory regulation or exceeds the permitted use, you will need to obtain permission directly from the copyright holder. To view a copy of this licence, visit http://creativecommons.org/licenses/by/4.0/.

\section{References}

1. Fong, D. Y. T., Wong, J. Y. H., \& Huang, L. (2018). Effect of noise tolerance on non-restorative sleep: A population-based study in Hong Kong. British Medical Journal Open, 8(3), e020518. https ://doi.org/10.1136/bmjopen-2017-020518.

2. Wilkinson, K., \& Shapiro, C. (2012). Nonrestorative sleep: Symptom or unique diagnostic entity? Sleep Medicine, 13(6), 561-569. https://doi.org/10.1016/j.sleep.2012.02.002.

3. Wakasugi, M., Kazama, J. J., Narita, I., Iseki, K., Moriyama, T., Yamagata, K., et al. (2014). Association between combined lifestyle factors and non-restorative sleep in Japan: A cross-sectional study based on a Japanese health database. PLoS ONE, 9(9), e108718. https://doi.org/10.1371/journal.pone.0108718.

4. Stone, K. C., Taylor, D. J., McCrae, C. S., Kalsekar, A., \& Lichstein, K. L. (2008). Nonrestorative sleep. Sleep Medicine Review, 12(4), 275-288. https://doi.org/10.1016/j.smrv.2007.12.002.

5. Ohayon, M. M. (2005). Prevalence and correlates of nonrestorative sleep complaints. Archives of Internal Medicine, 165(1), 35-41. https://doi.org/10.1001/archinte.165.1.35.

6. Roth, T., Jaeger, S., Jin, R., Kalsekar, A., Stang, P. E., \& Kessler, R. C. (2006). Sleep problems, comorbid mental disorders, and role functioning in the national comorbidity survey replication. Biological Psychiatry, 60(12), 1364-1371. https://doi.org/10.1016/j. biopsych.2006.05.039.

7. Zhang, J., Lam, S. P., Li, S. X., Li, A. M., \& Wing, Y. K. (2012). The longitudinal course and impact of non-restorative sleep: A five-year community-based follow-up study. Sleep Medicine, 13(6), 570-576. https://doi.org/10.1016/j.sleep.2011.12.012.

8. Chiu, H. Y., Wang, M. Y., Chang, C. K., Chen, C. M., Chou, K. R., Tsai, J. C., et al. (2014). Early morning awakening and nonrestorative sleep are associated with increased minor non-fatal accidents during work and leisure time. Accident Analysis \& Prevention, 71, 10-14. https://doi.org/10.1016/j.aap.2014.05.002.

9. Kawada, T. (2012). Feeling refreshed by sleep can predict psychological wellbeing assessed using the general health questionnaire in male workers: A 3-year follow-up study. Psychiatry Investigation, 9(4), 418-421. https://doi.org/10.4306/pi.2012.9.4.418.

10. Sarsour, K., Van Brunt, D. L., Johnston, J. A., Foley, K. A., Morin, C. M., \& Walsh, J. K. (2010). Associations of nonrestorative sleep with insomnia, depression, and daytime function. Sleep Medicine, 11(10), 965-972. https://doi.org/10.1016/j.sleep.2010.08.007.

11. Okamoto, M., Kobayashi, Y., Nakamura, F., \& Musha, T. (2017). Association between nonrestorative sleep and risk of diabetes: A cross-sectional study. Behavioral Sleep Medicine, 15(6), 483-490. https://doi.org/10.1080/15402002.2016.1163701.

12. Roth, T. (2010). What is the nature of nonrestorative sleep? Sleep Medicine, 11(10), 963-964. https://doi.org/10.1016/j.sleep .2010.11.001.

13. Wilkinson, K., \& Shapiro, C. (2013). Development and validation of the Nonrestorative Sleep Scale (NRSS). Journal of Clinical Sleep Medicine, 9(9), 929-937. https://doi.org/10.5664/ jesm.2996. 
14. Li, S., Fong, D. Y. T., Wong, J. Y. H., Wilkinson, K., Shapiro, C., Choi, E. P. H., et al. (2019). Nonrestorative sleep scale: reliable and valid for the Chinese population. Quality of Life Research. https://doi.org/10.1007/s11136-019-02134-8.

15. Roy, C., Bakan, G., Li, Z., \& Nguyen, T. H. (2016). Coping measurement: Creating short form of Coping and Adaptation Processing Scale using item response theory and patients dealing with chronic and acute health conditions. Applied Nursing Research, 32, 73-79. https://doi.org/10.1016/j.apnr.2016.06.002.

16. Cappelleri, J. C., Jason Lundy, J., \& Hays, R. D. (2014). Overview of classical test theory and item response theory for the quantitative assessment of items in developing patient-reported outcomes measures. Clinical Therapeutics, 36(5), 648-662. https://doi. org/10.1016/j.clinthera.2014.04.006.

17. Andersson, B., \& Xin, T. (2018). Large sample confidence intervals for item response theory reliability coefficients. Educational and Psychological Measurement, 78(1), 32-45. https://doi. org/10.1177/0013164417713570.

18. Buysse, D. J., Reynolds, C. F., 3rd, Monk, T. H., Berman, S. R., \& Kupfer, D. J. (1989). The Pittsburgh Sleep Quality Index: A new instrument for psychiatric practice and research. Psychiatry Research, 28(2), 193-213. https://doi.org/10.1016/01651781(89)90047-4.

19. Chong, A. M. L., \& Cheung, C. K. (2012). Factor structure of a Cantonese-version pittsburgh sleep quality index. Sleep and Biological Rhythms, 10(2), 118-125. https://doi.org/10.111 1/j.1479-8425.2011.00532.x.

20. Ye, Z. J., Liang, M. Z., Zhang, H. W., Li, P. F., Ouyang, X. R., Yu, Y. L., et al. (2018). Psychometric properties of the Chinese version of resilience scale specific to cancer: An item response theory analysis. Quality of Life Research, 27(6), 1635-1645. https ://doi.org/10.1007/s11136-018-1835-2.

21. Izquierdo, I., Olea, J., \& Abad, F. J. (2014). Exploratory factor analysis in validation studies: Uses and recommendations. Psicothema, 26(3), 395-400. https://doi.org/10.7334/psicothema 2013.349.

22. Dodeen, H., \& Al-Darmaki, F. (2016). The application of item response theory in developing and validating a shortened version of the Emirate Marital Satisfaction Scale. Psychological Assessment, 28(12), 1625-1633. https://doi.org/10.1037/pas0000296.

23. Costello, A. B., \& Osborne, J. W. (2005). Best practices in exploratory factor analysis: Four recommendations for getting the most from your analysis. Practical Assessment Research \& Evaluation, 10(7). Retrived from: https://pareonline.net/getvn .asp? $\mathrm{v}=10 \& \mathrm{n}=7$. Accessed 17 Aug 2019.

24. Xu, H., \& Tracey, T. J. G. (2017). Development of an abbreviated Career Indecision Profile-65 using item response theory: The CIPShort. Journal of Counseling Psychology, 64(2), 222-232. https ://doi.org/10.1037/cou0000182.

25. Reeve, B. B., Hays, R. D., Bjorner, J. B., Cook, K. F., Crane, P. K., Teresi, J. A., et al. (2007). Psychometric evaluation and calibration of health-related quality of life item banks: Plans for the Patient-Reported Outcomes Measurement Information System (PROMIS). Medical Care, 45(5 Suppl 1), S22-S31. https://doi. org/10.1097/01.mlr.0000250483.85507.04.

26. Edelen, M. O., \& Reeve, B. B. (2007). Applying item response theory (IRT) modeling to questionnaire development, evaluation, and refinement. Quality of Life Research, 16(Suppl 1), 5-18. https ://doi.org/10.1007/s11136-007-9198-0.

27. Jutten, R. J., Peeters, C. F. W., Leijdesdorff, S. M. J., Visser, P. J., Maier, A. B., Terwee, C. B., et al. (2017). Detecting functional decline from normal aging to dementia: Development and validation of a short version of the Amsterdam IADL Questionnaire. Alzheimers \& Dementia (Amsterdam, Netherlands), 8, 26-35. https://doi.org/10.1016/j.dadm.2017.03.002.
28. Peterson, E. R., Mohal, J., Waldie, K. E., Reese, E., Atatoa Carr, P. E., Grant, C. C., et al. (2017). A cross-cultural analysis of the infant behavior questionnaire very short form: An item response theory analysis of infant temperament in New Zealand. Journal of Personality Assessment, 99(6), 574-584. https://doi. org/10.1080/00223891.2017.1288128.

29. Masters, G. N. (2009). The analysis of partial credit scoring. Applied Measurement in Education, 1(4), 279-297. https://doi. org/10.1207/s15324818ame0104_2.

30. Baker, F. B. (2001). The basics of item response theory (2nd ed.). College Park: ERIC Clearinghouse on Assessment and Evaluation.

31. Reise, S. P., Widaman, K. F., \& Pugh, R. H. (1993). Confirmatory factor analysis and item response theory: Two approaches for exploring measurement invariance. Psychological Bulletin, 114(3), 552-566. https://doi.org/10.1037/0033-2909.114.3.552.

32. Cao, M., Tay, L., \& Liu, Y. (2017). A Monte Carlo study of an iterative Wald test procedure for DIF analysis. Educational and Psychological Measurement, 77(1), 104-118. https://doi. org/10.1177/0013164416637104.

33. Meade, A. W., \& Wright, N. A. (2012). Solving the measurement invariance anchor item problem in item response theory. Journal of Applied Psychology, 97(5), 1016-1031. https://doi.org/10.1037/ a0027934.

34. Chiesi, F., Morsanyi, K., Donati, M. A., \& Primi, C. (2018). Applying item response theory to develop a shortened version of the need for cognition scale. Advances in Cognitive Psychology, 14(3), 75-86. https://doi.org/10.5709/acp-0240-z.

35. Chio, F. H. N., Lai, M. H. C., \& Mak, W. W. S. (2018). Development of the Nonattachment Scale-Short Form (NAS-SF) using item response theory. Mindfulness, 9(4), 1299-1308. https://doi. org/10.1007/s12671-017-0874-z.

36. Allen, M. J., \& Yen, W. M. (2001). Introduction to measurement theory. Long Grove, IL: Waveland Press.

37. Harel, D., \& Baron, M. (2019). Methods for shortening patientreported outcome measures. Statistical Methods in Medical Research, 28(10-11), 2992-3011. https://doi.org/10.1177/09622 80218795187.

38. Revelle, W. (2020). psych: Procedures for psychological, psychometric, and personality research. Retrived from https://cran.r-proje ct.org/web/packages/psych/index.html. Accessed 23 Feb 2020.

39. Chalmers, P., Pritikin, J., Robitzsch, A., Zoltak, M., Kim, K., Falk, C. F., et al. (2019). mirt: Multidimensional item response theory. Retrived from https://mirrors.tuna.tsinghua.edu.cn/CRAN/. Accessed 26 Apr 2019.

40. Rizopoulos, D. (2018). ltm: Latent trait models under IRT. Retrived from https://mirrors.tuna.tsinghua.edu.cn/CRAN/. Accessed 26 Apr 2019.

41. Berkelaar, M., Konis, K., \& Schwendinger, F. (2020). lpSolveAPI: R Interface to 'lp_solve' version 5.5.2.0. Retrived from https:// cran.r-project.org/web/packages/lpSolveAPI/index.html. Accessed 23 Feb 2020.

42. An, X, Yung, Y-F., \& Institute, S. (2014). Item response theory: What it is and how you can use the IRT procedure to apply it. Retrived from https://support.sas.com/resources/papers/proce edings14/SAS364-2014.pdf. Accessed 7 July 2019.

43. Woods, C. M., Cai, L., \& Wang, M. (2012). The Langer-Improved Wald test for DIF testing with multiple groups: Evaluation and comparison to two-group IRT. Educational and Psychological Measurement, 73(3), 532-547. https://doi.org/10.1177/00131 64412464875

44. Fong, D. Y. T., Takemura, N., Chau, P. H., Wan, S. L. Y., \& Wong, J. Y. H. (2017). Measurement properties of the Chinese Weinstein noise sensitivity scale. Noise \& Health, 19(89), 193-199. https:// doi.org/10.4103/nah.NAH_110_16. 
45. Harel, D., Mills, S. D., Kwakkenbos, L., Carrier, M. E., Nielsen, K., Portales, A., et al. (2019). Shortening patient-reported outcome measures through optimal test assembly: Application to the Social Appearance Anxiety Scale in the Scleroderma PatientCentered Intervention Network Cohort. British Medical Journal Open, 9(2), e024010. https://doi.org/10.1136/bmjopen-2018024010.

46. Ishihara, M., Harel, D., Levis, B., Levis, A. W., Riehm, K. E., Saadat, N., et al. (2019). Shortening self-report mental health symptom measures through optimal test assembly methods:
Development and validation of the Patient Health QuestionnaireDepression-4. Depression and Anxiety, 36(1), 82-92. https://doi. org/10.1002/da.22841.

Publisher's Note Springer Nature remains neutral with regard to jurisdictional claims in published maps and institutional affiliations. 